\title{
The Mass Function of Newly Formed Stars (Review)
}

\author{
Lynne A. Hillenbrand ${ }^{1}$ \\ California Institute of Technology; MS 105-24; Pasadena, CA 91125 \\ lah@astro.caltech.edu
}

\section{Introduction}

The topic of the stellar "original mass function" has a nearly 50 year history, dating to the publication in 1955 of Salpeter's seminal paper. In this review I discuss the many more recent results that have emerged on the initial mass function (IMF), as it is now called, from studies over the last decade of resolved populations in star forming regions and young open clusters.

The spiral structure of our Galaxy can be traced over many kilo parsecs near the plane $\left(b<5^{\circ}\right)$ via indicators of imminent, ongoing, or recent star formation such as molecular gas, HII regions, and OB stars. Locally, however, many of these same diagnostics can be found far above the Galactic plane. The ring of local star formation, historically termed the Gould Belt (Gould 1879 ), is inclined by $\sim 20^{\circ}$ with respect to the plane, and extends as close as $150 \mathrm{pc}$ at its nearest and $750 \mathrm{pc}$ at its furthest. Towards the outer part of the Galaxy along this ring are the Perseus, Orion, and Monoceros clouds, crossing the plane in Cygnus/Cepheus, and towards the inner Galaxy the Ophiuchus, Scorpius, Centaurus, Lupus, and Chamaeleon clouds again crossing the plane in Vela. Notably the famed Taurus-Auriga star forming region is not part of the Gould's Belt but rather located in the vicinity of the inferred center of the expanding ring, near the PerOB3 groups and dispersed Cas-Tau association. The Gould's Belt phenomenon recently has been repopularized based on the nearby young $(<100 \mathrm{Myr})$ field stellar population uncovered by the Hipparcos and ROSAT all-sky surveys, as well as from new appreciation of the spatial distribution of young neutron stars, pulsars, and gamma ray sources. The fortuitous geometry of local star formation enables us to study stellar populations relatively free of the foreground / background contamination and severe crowding and confusion which dominate investigations of more distant Galactic plane star formation.

Looking in detail at an individual star-forming molecular cloud one finds that the stellar distribution is not random across the face of the cloud, but rather that young stars tend to congregate into small groups or aggregates and denser clusters. In Taurus, for example, there are distinct regions of nearsimultaneous aggregated star formation in addition to similarly aged stars sparsely spread across the cloud (e.g. Hartmann et al 2001). In Orion, which is a more massive molecular complex by a factor of $\sim 10$, the clusters (such as 
the Orion Nebula Cluster and NGC 2024) are denser but a similar dispersed population is still present (e.g. Strom, Strom \& Merrill 1993; Carpenter 2000). Summarizing the global clustering characteristics of the local regions of star formation, cluster densities range from unbound aggregates or groupings of tens of stars over a $0.1-0.5 \mathrm{pc}$ radius $\left(<10^{2} \mathrm{stars} / \mathrm{pc}^{3}\right)$ to possibly bound clusters containing hundreds to thousands of stars over a $0.5-5 \mathrm{pc}$ radius $\left(10^{3}-\right.$ $10^{5}$ stars $\left./ \mathrm{pc}^{3}\right)$. Furthermore, higher mass stars are associated with denser though not necessarily more populous clusters (Hillenbrand 1995; Testi et al. 1997). High-mass and low-mass stars do form in the same places and at the same times; drastically different star formation mechanisms, e.g. for the production of massive stars, apparently are not required. Given the wide dynamic range in mass of stars and indeed sub-stellar mass objects in star forming regions, such arenas are natural targets for studies of the IMF.

Investigation of the detailed form of the stellar/sub-stellar IMF in young clusters is motivated by the fact that the distribution of masses is one of the most basic characteristics of a stellar system. For a single region or cluster, a successful theory of star formation should predict the salient features of the IMF such as minimum / maximum masses and the location / width of any peak in the distribution. A related predisposition concerns the universality of the IMF. Molecular cloud fragmentation and star formation processes could lead to an IMF that depends on variables such as time, location, metallicity, local stellar density, cloud density or temperature, the pre-existence of massive stars, or whether star formation is stochastic or triggered (e.g. via external cloud compression). The detailed shape of the IMF is also important for several utilitarian reasons, particularly if the form is a universal one, including deduction of the past star formation rate in the Galaxy (increasing, decreasing, or constant in time), interpretation of integrated light from distant regions in our Galaxy or other galaxies, and constraining the fraction of baryonic mass tied up in low luminosity objects.

In advance of summarizing the large body of work on the IMF in star forming regions and young open clusters it is useful to call attention to pioneers of field star IMF studies, including Salpeter (1955), Miller \& Scalo (1979), Scalo (1986), Rana (1987), Kroupa, Tout \& Gilmore (1993), Kroupa (2001). The field IMF near the stellar/sub-stellar boundary has been explored by Reid et al. (1999) and Burgasser et al. (2001). High mass populations largely in clusters are summarized by Massey (1995). These papers include many analysis steps in transforming observational data to masses. First are the conversions of photometric and spectroscopic data to absolute magnitudes or luminosities and effective temperatures, and then the corrections for incompleteness, star formation histories, stellar evolution, and in some cases effects such as binarity. Useful are recent review articles on the IMF including those by lead authors Kroupa, Meyer, Elmegreen, Scalo, and Larson.

What we know from these field star studies is that the distribution of masses rises as $\mathrm{dN} / \mathrm{dM} \propto \mathrm{M}^{-\alpha}$ with the exponent $\alpha$ changing from $\sim 2.3$ 
at the highest masses (nearly identical to the original Salpeter result which probed masses $0.3-16 \mathrm{M}_{\odot}$ ) to $\sim 0.3$ near and across the sub-stellar boundary. While the slope does flatten towards lower masses, it should be emphasized that the IMF extends in a continuous manner from the stellar domain well into to the brown dwarf regime. As found both in the field and in star forming regions, there is nothing special about the hydrogen-burning limit in terms of the production rates of gravitationally bound objects from the ISM.

The various field star IMF's that have been proposed do have different implications for the average stellar mass and the mass fraction in low- / intermediate- / high-mass objects. For example, considering a stellar population which extends from $0.1-120 \mathrm{M}_{\odot}$, a Salpeter (1955) IMF predicts that $96 \%$ of the stars and $60 \%$ of the mass are found at masses $<1 M_{\odot}$, with a mean stellar mass of $0.35 \mathrm{M}_{\odot}$. The log-normal form of the Miller-Scalo (1979) IMF, on the other hand, predicts that $78 \%$ of the stars and $31 \%$ of the mass are found at masses $<1 M_{\odot}$ with a mean stellar mass of $0.89 \mathrm{M}_{\odot}$, while the multiple power law Kroupa (2001) prediction is that $90 \%$ of the stars and $45 \%$ of the mass are found at masses $<1 M_{\odot}$, with a mean stellar mass of $0.80 \mathrm{M}_{\odot}$. Extending the lower mass limit of the population through the brown dwarf domain to $0.01 \mathrm{M}_{\odot}$ implies for the Kroupa IMF that $37 \%$ of objects and $4 \%$ of the mass are found in brown dwarfs, and that the mean mass per object (star / brown dwarf) drops to $0.38 \mathrm{M}_{\odot}$. An additional way in which to think of the IMF as a probability distribution is to ask how many stars are expected in a cluster before a star of given mass is present. Again using the Kroupa multiple power-law slope one finds that 16 stars are needed before a $1 \mathrm{M}_{\odot}$ star is expected, 245 before the first $8 \mathrm{M}_{\odot}$ star is expected, and 8274 before a $120 \mathrm{M}_{\odot}$ object is expected. Drastically different numbers result from the various proposed IMF's and the implications are not inconsequential for calculations of quantities such as the number of ionizing photons or the number of proto-supernovae in a cluster.

\section{Advantages and Disadvantages of Studying the IMF in Star Forming Regions and Young Open Clusters}

In contrast to the field star studies, investigations of youthful environments have the advantage that all stars formed in the vicinity are still present; none have evolved into stellar remnants and none have escaped the gravitational grasp of the molecular cloud and/or dense stellar cluster. Thus what is measured is not only the present day mass function but the initial mass function as well, with no corrections needed. The IMF can thus be measured over a large dynamic range, ideally from $>100 \mathrm{M}_{\odot}$ down to $<0.08 \mathrm{M}_{\odot}$ in a single region. Importantly, young low mass stars and brown dwarfs are several (3-5) orders of magnitude more luminous at 1-10 Myr of age than they are on the main sequence, compressing the mass-luminosity relationship. Further, forming sub-stellar populations are much warmer than those 
in the solar neighborhood field which have been cooling for many Gyr. At 1 Myr of age any object with spectral type later than M6-M7 (i.e. $\mathrm{T}<3000$ $\mathrm{K})$ is a brown dwarf $\left(<0.08 \mathrm{M}_{\odot} \approx 80 \mathrm{M}_{\text {Jupiter }}\right)$ and by mid-L has mass $<10 \mathrm{M}_{\text {Jupiter }}$ according to most current pre-main sequence contraction models. Another advantage of young cluster populations for IMF studies is that individual regions are co-eval, equi-distant, and have the same metallicity. Finally, presence of molecular gas can screen against contaminating background stars, making membership easier to assume. Results from one region can be compared directly to those from another with perhaps different total mass, stellar density, or metallicity, and also to those from the solar neighborhood field which represents a mix of star formation episodes occurring across kpc of space and Gyr of time in our Galaxy.

However, there are also several disadvantages to working in youthful environments. These include the poorly calibrated evolution of the massluminosity relationship through the pre-main sequence stage, the presence of large and spatially variable extinction in star-forming regions, the possibility of photometric excesses due to processes associated with circumstellar (accretion) disks, the spatial crowding and nebulosity which complicate observations, and finally the lack of truly superb statistics for individual regions.

\section{Methodology for IMF Study of Young Stars}

With the above benefits and limitations in mind, various techniques have been developed in attempting to measure the IMF in star-forming regions and young open clusters. These include the use of luminosity functions and a model of the star formation history (e.g. Zinnecker, Rieke, Comeron, Wilking, Strom, Lada, Alves, Muench), employment of color-magnitude diagrams and a photometric de-reddening technique as well as a model of the star formation history (e.g. Meyer, Carpenter, McCaughrean, Figer), and finally the construction of Hertzsprung-Russell diagrams which require both spectroscopy and photometry (e.g. Herbig, Strom, Hillenbrand, Brown, Luhman, Preibisch, Rebull). All of these methods must assume a set of theoretical mass-luminosity relationships and translate them into the needed format for comparison to the data.

The spectroscopic techniques are the most robust, but consequently require the most observational and analytical effort. Feasibility with large telescopes $(8-10 \mathrm{~m})$ extends well into the sub-stellar regime for the more proximate $(<500 \mathrm{pc})$ regions of recent star formation, but the technique is applicable only to the upper end of the IMF in more distant ( $>1-2 \mathrm{kpc})$ regions. Spectroscopy is usually performed in the V, I, J, or K bands $(0.5-2 \mu \mathrm{m})$ and enables the photospheric temperature to be estimated through adoption of a spectral-type to effective temperature relationship. Spectral types are derived from ratios of specific atomic lines or molecular bands with the most diagnostic features generally found in the spectral regime closest to the peak 
wavelength of the stellar blackbody. The calibrations to effective temperature are established through fundamental techniques such as the infrared flux method, measured diameters, or for intrinsically less luminous objects via theoretical modelling of stellar atmospheres. Once a spectral type is determined an intrinsic color scale is used to compare observed to expected (given the spectral type) colors and thereby derive the color excess. This can be converted using an adopted extinction law into equivalent V-band extinction. De-reddened photometry is then combined with a bolometric correction scale to produce bolometric luminosities. Finally, the derived effective temperature and bolometric luminosity are compared to those predicted from pre-main sequence evolutionary models to infer stellar masses and ages.

It should not go without mention that the pre-main sequence evolutionary models (e.g. those by D'Antona \& Mazzitelli 1997; Swenson et al. 1994; Baraffe et al. 1998; Siess et al. 2000; Palla \& Stahler 2000; Yi et al. 2003) remain poorly calibrated. Variations between these predictions can be as much as factors of 2 in mass and factors of 3 in age. In most cases such models combined with quantities derived from observational data such as HertzsprungRussell diagrams, color-magnitude diagrams, or luminosity functions are the only means available for inferring stellar masses. However, fundamentally determined masses are those calculated from observations of dynamical systems such as eclipsing double-line spectroscopic binaries or, to lower precision, systems surrounded by circumstellar disks whose keplerian rotation can be measured. Comparisons between the (limited) available dynamical masses and pre-main sequence evolutionary tracks demonstrate that most existing sets of tracks systematically under-predict low-mass pre-main sequence masses by up to $20-30 \%$ (Hillenbrand \& White 2004).

\section{IMF Results in Youthful Regions}

Before summarizing and synthesizing the results on stellar/sub-stellar IMFs in star-forming regions and young open clusters over the past decade, I call attention to a few recent results involving my own collaborators as examples of the sort of work being done. First at the high-mass end, Slesnick, Hillenbrand, \& Massey (2002) performed a UBV photometric + BV-band spectroscopy survey of the $\mathrm{H} / \chi$ Per region, finding a typical Salpeter-like IMF for each of the two clusters $(\Gamma=-1.36 \pm 0.20$ and $\Gamma=-1.25 \pm 0.23)$ over the mass range $4-40 \mathrm{M}_{\odot}$ with $\sim 40$ past supernova expected. At the other end of the mass spectrum, Slesnick, Hillenbrand, \& Carpenter (2004) have studied the sub-stellar domain of the Orion Nebula Cluster using JHK photometry and $\mathrm{J} / \mathrm{K}$ spectra, confirming the turnover suspected by several authors based on analyses at higher masses (Hillenbrand 1997; Luhman 2000; Muench et al 2002). Depending on the behavior of the mass-luminosity-temperature relationships - which are still highly uncertain at such low masses - there may even be a flattening or potential rise towards the lowest mass brown dwarfs. 
Generalizing now to the local set of star forming regions and young open clusters, it is often the case that incompleteness and overall poor statistics preclude robust measurement of the stellar/sub-stellar IMF. Assembly of existing literature demonstrates that close to 5000 stars in 24 regions can be located on the HR diagram and compared to theoretical evolutionary tracks. However, only a few regions are reasonably complete into the brown dwarf regime, namely the Orion Nebula Cluster, IC 348, and the Pleiades. These regions compare well to a log-normal type (Miller-Scalo 1979) mass function with acceptable statistical and systematic (due to methodology) variation.

To provide a meaningful assessment of the IMF, observers need to measure its shape in detail, including the dynamic range, peak, and width of the peak. Attempts at functional fits are welcome. At present, however, most authors simply force single power laws through their data and quote the resulting best-fit slope, sometimes even with an errorbar. Taken en ensemble, however, these forced power laws present a very different picture of the IMF. Figure 1 is a plot inspired by similar graphs presented by Scalo (1998) and Kroupa (2001) of the slope of the initial mass function $\Gamma$ where $d N(\log M) / d \log M \propto M^{\Gamma}$ and $d N(M) / d M \propto M^{-\alpha}$ such that $\Gamma=1-\alpha$, shown as a function of $\langle M\rangle / M_{\odot}$ where $\langle M\rangle$ is the average mass over which a common slope is claimed to apply. The data are drawn from an extensive list of IMF primary references appearing over the last decade. Many different techniques are assimilated, including spectroscopic investigations, color-magnitude diagrams, and luminosity functions. Similar plots of $\Gamma$ as a function of minimum mass or maximum mass, rather than the average mass, are also instructive. What is clear from these Figures is that at the high mass end $\left(\mathrm{M}>2-3 \quad M_{\odot}\right)$ reported IMF slopes are constant as a function of mass and distributed around the original Salpeter value of $\Gamma=-1.35$. At lower masses $\left(\mathrm{M}<1-2 M_{\odot}\right)$, however, the reported slopes deviate from Salpeter in a manner such that the IMF slope continually becomes shallower / flatter towards lower masses.

There scatter in Figure 1 amounts to $\sim 1$ dex for any mean mass. Although it may be tempting to interpret this scatter as real variation in the IMF, there are several affects which may in part explain it. As stated above there are differences in methodology between authors, and while spectroscopic investigations are indeed the most robust, they are not practical for all regions; of note is that there are no systematic differences in Figure 1 between the spectroscopic investigations and those that are purely photometric (colormagnitude diagrams or luminosity functions). Another possible effect is that of systematics present in the different effective temperature or bolometric correction scales adopted by different authors, but again, offsets between authors or groups are not evident in the results as presented in the format of Figure 1. The adopted set of pre-main sequence theory is yet another way to introduce systematic differences. Finally, the statistical robustness of the results should be considered in lieu of generally small sample sizes, particu- 


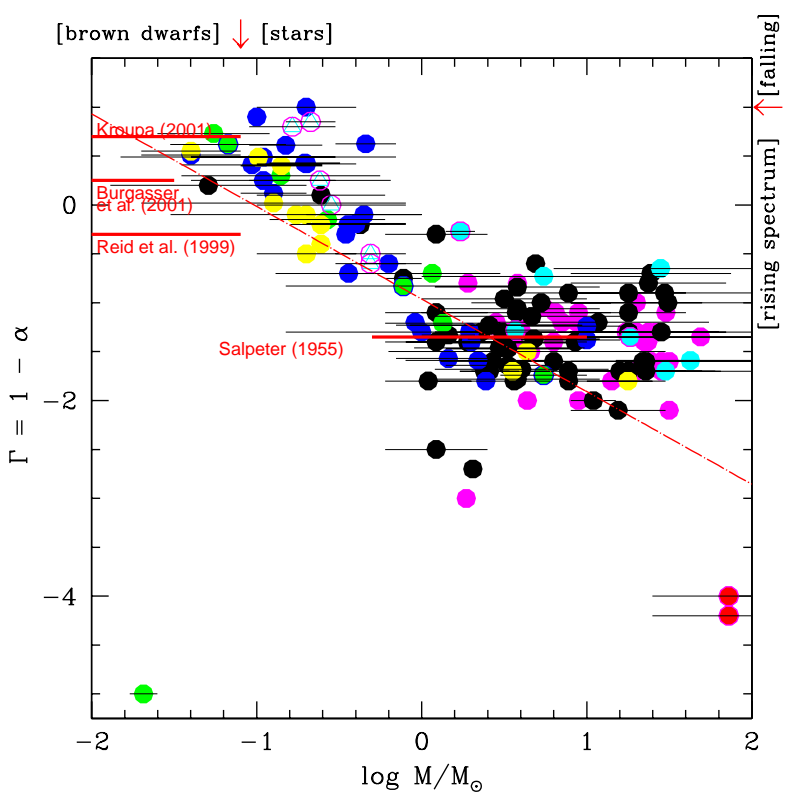

Fig. 1. Values quoted in the literature for the slope of the initial mass function plotted against mean mass (points), with the range of masses over which the single slope is claimed to apply indicated (horizontal lines). Filled circles represent star-forming regions and young open clusters while triangles are globular clusters. Continual change of the mass function slope with mean mass below about $1 \mathrm{M}_{\odot}$ demonstrates that a quadratic or log-normal function best describes the composite data at low masses. Above several $\mathrm{M}_{\odot}$ the scatter is more considerable, and the quadratic or log-normal function yields to a flatter slope that averages close to the Salpeter (1955) value. The dash-dot line shows a Miller-Scalo (1979) log-normal.

larly at the high mass end where by the very nature of the IMF the poisson statistics are the poorest. Kroupa (2001) has investigated this last point by simulating a version of Figure 1 using model populations differing in total numbers of stars $\left(10^{2}-10^{6}\right)$ sampled many times. The observed scatter about the Salpeter slope can in fact be reproduced through sampling error alone. Kroupa (2001) also investigates the scatter introduced by dynamical evolution of clusters on time scales of a few Myr to tens of Myr, and concludes that some of the scatter may be attributed to the redistribution of mass within or exterior to the gravitational bounds of a cluster.

In summary, the general trends emerging from the literature are for a universal IMF with a single Salpeter-like slope above masses of 1-3 $M_{\odot}$ and a constantly flattening slope towards lower masses, extending smoothly and continuously from low mass stars into the sub-stellar regime. At present, there is little evidence for substantial variations in the IMF from region to region, or over time. The scatter present in Figures of IMF slope vs mass are 
explainable by a combination of methodology differences, theory differences, and sampling statistics. Yes, there are outliers in such plots, and the regions in question should be investigated further to determine the accuracy of the reported results. But the dominant trends remain.

\section{Future Goals of IMF Studies}

Having crossed the sub-stellar boundary over the past few years, the next major threshold for low mass IMF studies to reach is the theoretical limit for fragmentation out of a molecular cloud, derived by calculating the ability of a Jeans' mass / radius object contracting on the free-fall time to radiate its own gravitational energy. Thus far no low-mass cut-off in the IMF has been observed. Observationally, the Next Generation Space Telescope (now JWST) should be sensitive at the distance and age of the Orion Nebula Cluster, for example, to objects as low in mass as $1 \mathrm{M}_{\text {Jupiter }}$. Given an appropriate means of sorting between field star contaminants and cluster members at such faint magnitudes, the goal of determining the lowest free-forming mass object should be reached in this dense and populous star forming region.

Another major question concerns the universality of the IMF. Is the mass spectrum the same everywhere, that is, invariant with respect to time, location, size or density of the star-forming environment, metallicity, other initial conditions such as temperature or pressure? Can we relate the IMF to some physical aspect of the star formation process? Thus far no systematic variations have been detected, for example between high-mass star forming environments of varying metallicity (e.g. Massey et al. 1995ab) or between low-mass star forming environments of different stellar density (e.g. Luhman 2000), or even in "extreme" environments such as R 136 (Massey \& Hunter 1998) in the Large Magellenic Cloud. That is not to say that no such trends are present. Rather, to within our ability to provide robust statistical assessment, variations have not yet been detected. A very few regions with "unusual" IMFs have been presented (e.g. the Hillenbrand et al. 1995 discussion of the bias of the $\mathrm{BD}+404124$ region towards higher mass stars and the Briceno et al. 2002 presentation of the lack of brown dwarfs in Taurus) though whether these regions are truly unusual or merely the expected statistical outliers remains unquantified. A goal then is to more rigorously quantify the invariance of the IMF.

\section{Summary}

- The IMF is not a single power law but rather continually changes its slope below masses of 2-3 $\mathrm{M}_{\odot}$.

- There is nothing special about the hydrogen burning limit in terms of the frequency distribution of masses above or below this boundary. 
- Thus far no low-mass cut off has been observed in the IMF, with limits reaching perhaps as low as 20-30 $\mathrm{M}_{\text {Jupiter }}$ in some star formation regions. The next fundamental boundary is that of opacity-limited fragmentation.

- The lack of strong evidence for IMF variations suggest that its functional form may be universal and therefore independent of any of the physical variables suspected to play a role in the star formation process.

\section{References}

1. I. Baraffe, G. Chabrier, F. Allard, and P.H. Hauschildt, AA, 337, 403 (1998)

2. C. Briceno, K.L. Luhman, L. Hartmann, J.R. Stauffer, and J.D. Kirkpatrick, ApJ, 580, 317 (2002)

3. A.J. Burgasser PhD Thesis, California Institute of Technology (2001)

4. J.M. Carpenter AJ 120, 3139 (2000)

5. F. D'Antona, and I. Mazzitelli, in Cool stars in Clusters and Associations, ed. R. Pallavicini, \& G. Micela, Mem. S. A. It., 68, 807 (1997)

6. B.A. Gould: Uranometria Argentina, ed. P.E. Corni, Buenos Aires, p. 354 (1879)

7. L. Hartmann, J Ballesteros-Paredes, and E.A Bergin ApJ 562, 852 (2001)

8. L.A. Hillenbrand PhD Thesis, University of Massachusetts (1995)

9. L.A. Hillenbrand, AJ, 113, 1733 (1997)

10. L.A. Hillenbrand and R.J. White, ApJ, in press (2004).

11. L.A. Hillenbrand, M.R. Meyer, S.E. Strom, and M.F. Skrutskie, AJ 109, 280 (1995)

12. P. Kroupa, MNRAS 322, 231 (2001)

13. P. Kroupa, MNRAS 322, 231 (2001)

14. P. Kroupa, C.A. Tout, and G. Gilmore, MNRAS 262, 545 (1993)

15. K.L. Luhman, G.H. Rieke, E.T. Young, et al., ApJ, 540, 1016 (2000)

16. P. Massey and D.A. Hunter, ApJ 493, 180 (1998)

17. P. Massey, K.E. Johnson, and K. Degioia-Eastwood, ApJ 454, 151 (1995a)

18. P. Massey, C.C. Lang, K. Degioia-Eastwood, and C.D. Garmany, ApJ 438, $188(1995 \mathrm{~b})$

19. G.E. Miller and J.M. Scalo, ApJS 41, 513 (1979)

20. F. Palla, and S.W. Stahler, ApJ, 525, 772 (1999)

21. N.C. Rana, AA, 184, 104 (1987)

22. I.N. Reid, J.D. Kirkpatrick, J. Liebert, et al., ApJ, 521, 613 (1999)

23. E.E. Salpeter ApJ, 121, 161 (1955)

24. J.M. Scalo, Fund. Cos. Phys. 11, 1 (1986)

25. J.M. Scalo, The Stellar Initial Mass Function (38th Herstmonceux Conference), edited by G. Gilmore and D. Howell, ASP Conf. Ser. 142, 201 (1998)

26. L. Siess, E. Dufour, and M. Forestini, AA, 358, 593 (2000)

27. C.L. Slesnick, J.M. Carpenter, and L.A. Hillenbrand, ApJ, submitted (2004)

28. C.L. Slesnick, L.A. Hillenbrand, and P. Massey, ApJ, 576, 880 (2002)

29. K.M. Strom, S.E. Strom, and K.M. Merrill ApJ 412, 233 (1993)

30. F.J. Swenson, J. Faulkner, F.J. Rogers, and C.A. Iglesias ApJ, 425, 286 (1994)

31. L. Testi, F. Palla, T. Prusti, A. Natta, and S. Maltagliati, AA 320, 159 (1997)

32. S. Yi, Y.-C. Kim, and P. Demarque, ApJS, 144, 259 (2003) 